\title{
Comparison of Results Following Modified Widman Flap Surgery With and Without Surgical Dressing
}

\author{
Donald R. Allen and Raul G. Caffesse†
}

Accepted for publication 26 October 1983

\begin{abstract}
THE CLINICAL EFFECTS of a periodontal dressing after modified Widman flap surgery were studied. A total of thirty surgical sites in 13 patients were included in the study. Half of the sites were not covered by a periodontal dressing while the other half were. To effect a blind evaluation, the dressings were placed and removed by someone other than the examiner. Gingival crevicular fluid flow and gingival inflammation were measured prior to surgery, 2 weeks, 1 month and 2 months after surgery. Clinical attachment level and pocket depth were measured prior to surgery, 1 month and 2 months after surgery. All patients were instructed in oral hygiene procedures and received rubber cup prophylaxes and flossing at 1 week, 2 weeks, 1 month and 2 months postoperatively. At the conclusion of the study, a questionnaire was administered to each patient. No significant differences were found between dressed and undressed segments regarding changes in clinical attachment levels, pocket depth, or gingival inflammation. The omission of a dressing did not result in a greater incidence of discomfort. More patients stated preference for no dressing.
\end{abstract}

Periodontal dressings have been used routinely following surgical procedures in periodontics primarily to provide protection of the wound and to aid in comfort during mastication. Recently the value of postsurgical dressings has been questioned. ${ }^{1,2}$ There is controversy as to the effect of periodontal dressings upon the quality of healing. Consequently, some clinicians routinely omit postsurgical dressings provided good flap adaptation is achieved. The purpose of the present study is to evaluate clinically the quality of healing following the modified Widman flap with and without the use of postsurgical dressing.

\section{MATERIALS AND METHODS}

Thirteen patients, four males and nine females, average age of 46 years (ranging from 24 to 59), who were scheduled for surgery at the University of Michigan Department of Periodontics, were selected for the study. The patients were free of systemic disease and taking no medications. Each patient provided at least two surgical sites and in some instances four surgical sites were utilized. Either the contralateral or opposing site in the maxilla and the mandible contained at least three teeth. A total of 30 surgical sites with similar periodontal involvement were compared. Only patients requir-

* Present address: 1259 N Kingshighway, St. Louis, MO 63112.

$\dagger$ Professor and Chairman, Department of Periodontics, University of Michigan School of Dentistry, Ann Arbor, MI 48109. ing modified Widman flap surgery were included in the study.

Prior to surgery the hygienic phase of therapy was completed. The hygienic phase included removal of overhanging restorations, scaling, root planing, polishing, any emergency treatment and oral hygiene instructions. Also, prior to any surgical treatment a consent form was explained to and signed by the patient. Surgeries were performed at least 1 month after completion of the hygienic phase.

Graduate students performed all surgical procedures, with the same operator treating both surgical sites to be compared. Special care was taken to achieve good flap adaptation. The experimental segment was decided by the "toss-of-the-coin" method. This segment was not covered by a periodontal dressing, while the control segment received a dressing (Perio Putty $\ddagger$ ). To effect a blind evaluation, the dressings were placed and removed 1 week postoperatively by someone other than the examiner. Neither antibiotics nor any other medications were used at the surgery sites.

The parameters used to assess clinical healing were crevicular fluid flow, gingival inflammation, pocket depth and attachment level. Further, a questionnaire was given to the patient at the completion of the experiment. All recordings were taken prior to surgery then 2 weeks, 1 month and 2 months after surgery.

$¥$ Cadco Dental Products, Los Angeles, CA. 
Table 1

Mean Differences in Attachment Level at Different Observation Periods Pairwise t-test*

\begin{tabular}{lccccccc}
\hline$\quad \mathrm{N}=15$ & Baseline & 1 Month & Difference $\dagger$ & Sign. & 2 Months & Difference $\dagger$ & Sign. \\
\hline Buccal & & & & & & & \\
$\quad$ Undressed & 2.63 & 2.97 & -0.34 & NS & 3.07 & -0.44 & NS \\
$\quad \begin{array}{l}\text { Dressed } \\
\text { Lingual }\end{array}$ & 2.87 & 3.30 & -0.43 & & 3.37 & -0.50 & \\
$\quad \begin{array}{l}\text { Undressed } \\
\quad \text { Dressed }\end{array}$ & 3.50 & 3.73 & -0.23 & NS & 3.57 & -0.07 & NS \\
$\begin{array}{l}\text { Interproximal } \\
\quad \text { Undressed }\end{array}$ & 3.40 & 4.17 & -0.77 & & 3.97 & -0.56 & \\
$\quad$ Dressed & 4.19 & 4.20 & -0.26 & NS & 4.18 & -0.24 & NS \\
\hline
\end{tabular}

* Measured in $\mathrm{mm}$ from cemento-enamel junction.

$\uparrow$ Denotes loss of attachment from baseline.

Crevicular fluid flow was measured with Periotron ${ }^{(n)}$.* Recordings were taken on the buccal and mesial aspects of the teeth.

Gingival inflammation was scored according to the Gingival Index of Löe and Silness. ${ }^{3}$ The scores for each tooth aspect were added and divided to obtain the Gingival Index for that particular tooth. The gingival indices for the teeth in each segment were added and divided to obtain the Gingival Index for that particular segment. The Gingival Index of that segment was then compared with the Gingival Index of either the contralateral or opposing segment.

Pocket depth was measured prior to surgery, 1 month and 2 months after surgery. Measurements were recorded at six different points on each tooth included in study. The measurements were taken at the mid-, mesial- and distal-buccal as close to the contact as possible, the mid, mesial- and distal-lingual as close to the contact as possible. All measurements were taken with the Hu-Friedy $\mathrm{CP}-11$ probe.

Attachment level was measured at the same intervals and on the locations on the teeth as pocket depth. It was measured from the base of the pocket to a fixed reference point on the tooth, preferably the cementoenamel junction. However, when restorations extended beyond the cementoenamel junction, their cervical margins were used as fixed reference points.

All patients were instructed by the operator to begin oral hygiene procedures, brushing and flossing, 12 to 24 hours postoperative in the nondressed segments and immediately after dressing removal in the dressed segments. Supervised oral hygiene procedures were performed 1 week, 2 weeks, 1 month and 2 months postoperatively following the recording of the clinical measurements. These oral hygiene procedures consisted of rubber cup prophylaxes and dental floss. At the conclusion of the study, a questionnaire was administered to each patient.

Statistical Analysis. Calibration and reproducibility testing for the different parameters used were performed prior to the study. Two randomly selected teeth in each

\footnotetext{
* Harco Electronics Limited, Winnipeg, Canada.
}

of three subjects with periodontal disease were scored three times each, not consecutively, to determine examiner variability.

The data collected were processed through the Michigan Terminal System. The MIDAS program was used in analyzing the data. The pairwise $t$-test was utilized to determine if any differences existed between the dressed and undressed segments. Mean measurements were computed at baseline, 2 weeks, 1 month and 2 months for the various parameters. Mean differences were computed from baseline unless otherwise indicated. The level of significance chosen was 0.05 .

\section{RESULTS}

Calibration. A repeated measurement analysis of variance was done to assess the reliability of the attachment level and pocket depth measurements. These measurements were repeated three times at each of the six sites on two teeth for three of the patients. The mean square error for attachment level was 0.64 and for pocket depth 0.30 . These mean square errors estimate the variance among the repeated measurements of attachment level and pocket depth respectively.

Baseline. Dressed and undressed areas were compared at baseline for all four parameters tested and found to be statistically similar at the 0.05 level of significance.

Attachment Level. The mean measurements of attachment level at different times of observation are shown in Table 1 and represented in Figure 1. An insignificant loss of attachment was recorded throughout the experiment. This loss was slightly greater buccally and lingually in the dressed segments.

Pocket Depth. The mean measurements of pocket depth are shown in Table 2 and Figure 2. Two-months observation showed an overall reduction in pocket depth. The reduction was greater for the dressed segments but was statistically insignificant.

Gingival Inflammation. The mean measurements of gingival inflammation are shown in Table 3 and Figure 3. At 2 weeks the undressed segments showed a decrease in gingival inflammation while the dressed segments showed a slight increase. At 1 month the measurements 
were reversed, and the differences from baseline were statistically significant $(P<0.05)$. At 2 months both the dressed and undressed segments showed a decrease below baseline value. The gingival inflammation at 2 months was slightly less for the dressed segments but this difference was statistically insignificant.

Gingival Crevicular Fluid. The mean measurements of gingival crevicular fluid are shown in Table 4 and Figure 3. At 2 weeks there was an increase in gingival crevicular fluid flow (GCF) over the baseline for both the undressed and dressed segments. At 1 month there

CHANGES IN ATTACHMENT LEVEL

\section{OUER TIME}

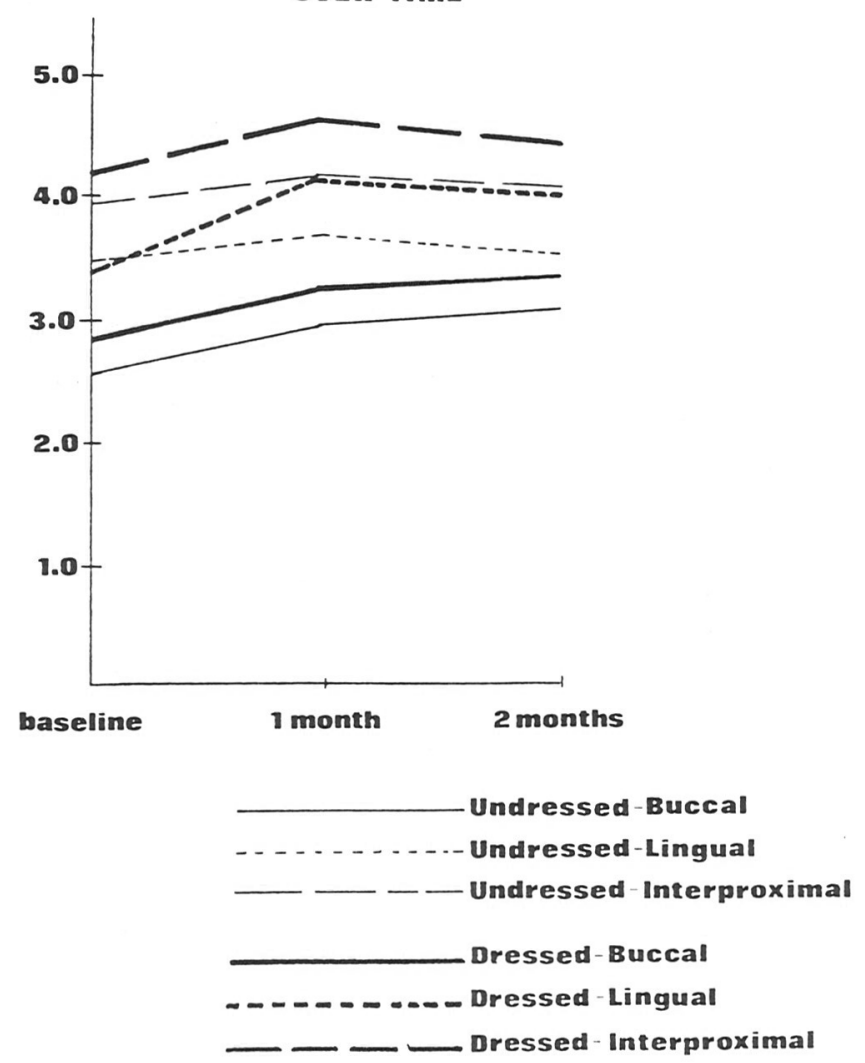

Figure 1. Changes in clinical attachment levels over time, in dressed and undressed areas after modified Widman flaps. was a decrease in GCF flow below baseline values for both the undressed and dressed segments. The decrease in GCF flow was greater in the dressed segments. However, this decrease was not statistically significant. At 2 months there was a further reduction in GCF flow in both the undressed and dressed segments. The undressed segments showed a slightly greater reduction in GCF flow than the dressed areas, but it was not statistically significant.

Questionnaire. The patients' experience during the 7-day postoperative period with regard to discomfort

\section{CHANGES IN POCKET DEPTH}

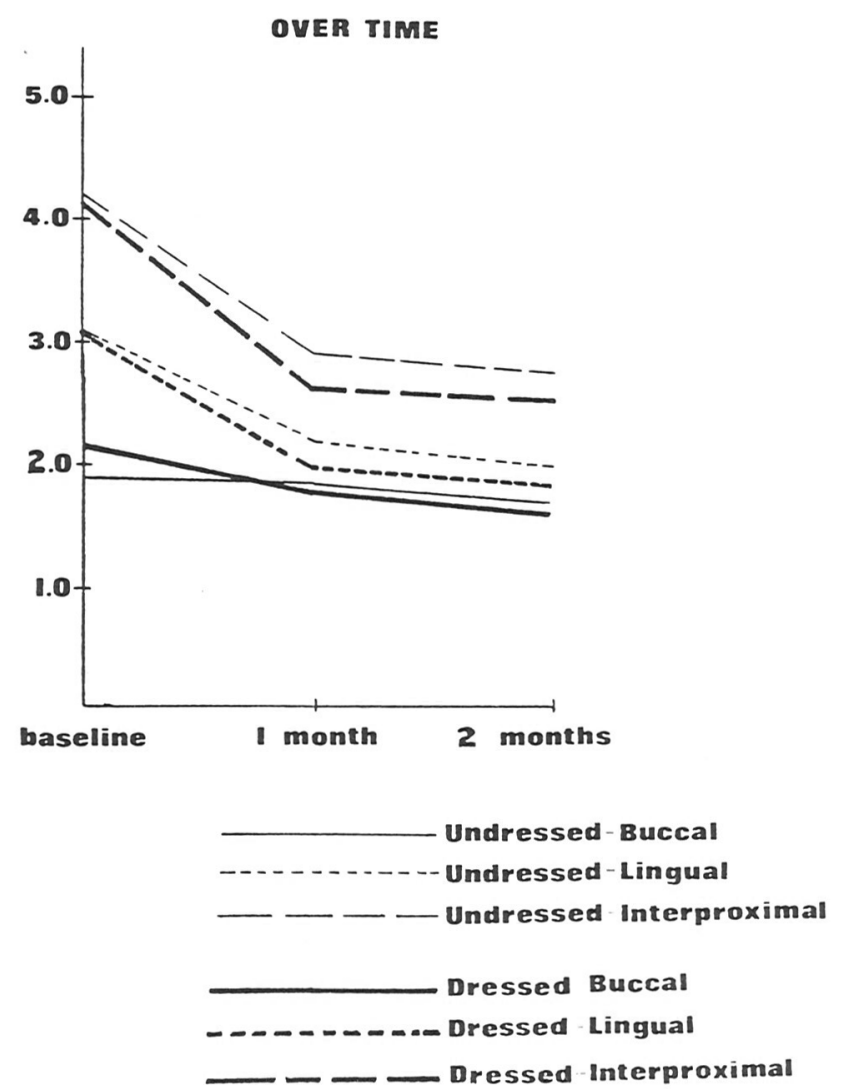

Figure 2. Changes in pocket depth over time in dressed and undressed areas after modified Widman flaps.

Table 2

Mean Differences in Pocket Depth at Different Observation Periods Pairwise t-test*

\begin{tabular}{|c|c|c|c|c|c|c|c|c|}
\hline$N=15$ & Baseline & 1 Month & $\begin{array}{l}\text { Differ- } \\
\text { ence }\end{array}$ & Sign. & 2 Months & $\begin{array}{l}\text { Differ- } \\
\text { ence }\end{array}$ & $\begin{array}{l}\text { Differ- } \\
\text { ence } \dagger\end{array}$ & Sign. \\
\hline \multicolumn{9}{|l|}{ Buccal } \\
\hline Undressed & 1.93 & 1.83 & 0.10 & \multirow{2}{*}{ NS } & 1.60 & 0.33 & 0.23 & \multirow{2}{*}{ NS } \\
\hline Dressed & 2.20 & 1.77 & 0.43 & & 1.67 & 0.53 & 0.19 & \\
\hline \multicolumn{9}{|l|}{ Lingual } \\
\hline Undressed & 3.13 & 2.33 & 0.80 & \multirow{2}{*}{ NS } & 2.03 & 1.10 & 0.30 & \multirow{2}{*}{ NS } \\
\hline Dressed & 3.10 & 2.03 & 1.07 & & 1.83 & 1.27 & 0.20 & \\
\hline \multicolumn{9}{|l|}{ Interproximal } \\
\hline Undressed & 4.18 & 2.97 & 1.21 & \multirow{2}{*}{ NS } & 2.82 & 1.36 & 0.15 & \multirow{2}{*}{ NS } \\
\hline Dressed & 4.16 & 2.75 & 1.40 & & 2.61 & 1.55 & 0.15 & \\
\hline
\end{tabular}

* Measured in $\mathrm{mm}$ from free gingival margin to base of crevice; $P>0.05$.

† Difference from 1 to 2 months. 
Table 3

Mean Differences in Gingival Inflammation at Different Observation Periods Pairwise t-test

\begin{tabular}{lcccccccccc}
\hline $\mathrm{N}=15$ & Baseline & 2 Weeks & Diff.* & Sign. & 1 Month & Diff.* & Sign. & 2 Months & Diff.* & Sign. \\
\hline Undressed & 1.05 & 1.01 & $-0.03 \dagger$ & \multirow{2}{*}{ NS } & 1.06 & $+0.01 \ddagger$ & \multirow{2}{*}{0.04} & 1.01 & $-0.04 \dagger$ & NS \\
Dressed & 1.05 & 1.06 & $+0.01 \ddagger$ & & 1.00 & $-0.05 \dagger$ & & 1.00 & $-0.05 \dagger$ & \\
\hline
\end{tabular}

* Differences from baseline.

$\dagger$ Denotes decrease in inflammation.

$\ddagger$ Denotes increase in inflammation.

CHANGES IN GI AND GCF OUER TIME
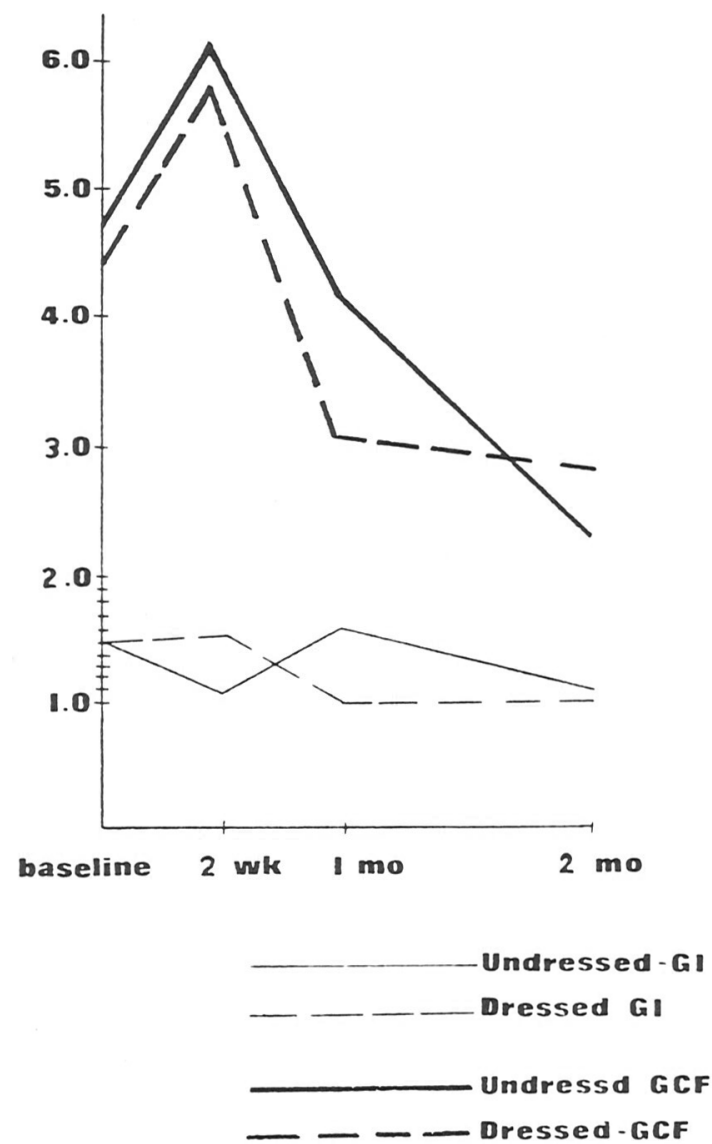

Figure 3. Changes in Gingival Index and gingival crevicular fluid flow in dressed and undressed areas after modified Widman flaps.

and preference for dressing or no dressing is given in Table 5. One third of the patients experienced more general discomfort without the dressing, while one third experienced more with the dressing and in one third the amount of discomfort was the same. Twenty per cent of the patients experienced more discomfort upon eating without the dressing and $20 \%$ experienced more with the dressing. In $60 \%$ of the patients the level of discomfort experienced was the same regardless of whether a dressing was used or not. The majority of the patients, $60 \%$, stated a preference for no dressing while only $40 \%$ stated a preference for the dressing.

\section{DISCUSSION}

The results from this clinical evaluation indicate that there is no difference in the clinical healing of a modified Widman flap whether or not a dressing is applied.

The finding that pocket depth measurements for the undressed segments exceeded that for the dressed segments is in agreement with Greensmith and Wade, ${ }^{1}$ although in the present study the difference was not significant. Jones and Cassingham ${ }^{2}$ found deeper pocket depths in the undressed segments at 6 weeks; however, by the 16th week the results were reversed. In the present study, a greater reduction in pocket depth occurred from 1 to 2 months in the undressed segments. Results similar to those of Jones and Cassingham might have occurred provided the length of the study would have been extended.

Attachment level was not a parameter used in previous studies comparing the effect on healing of dressings vs no dressings. ${ }^{1,2}$ In the present study, both the lingual and interproximal areas exhibited a slight but statistically insignificant gain in attachment from 1 month to 2 months. This occurred for both the dressed and undressed segments. These findings are consistent with the short term results (4-6 weeks) reported from the Michigan longitudinal study. ${ }^{4}$ The initial loss of attachment and subsequent gain probably reflects the lack of initial tissue maturation followed by increased maturation with time.

Gingival crevicular fluid measurements were obtained with the Periotron. This technique seems to be advantageous since less time is required for collection and it may eliminate examiner and evaporation error. ${ }^{5}$ Several studies have indicated a correlation between the amount of gingival crevicular fluid flow and the severity of clinical gingival inflammation. ${ }^{6}$ In the present study gingival crevicular fluid flow did not follow a pattern similar to the variation in Gingival Index. Gingival inflammation for the undressed segments was decreased at the 2 week interval while gingival crevicular fluid was increased. At 1 month gingival inflammation for the undressed segments was increased while crevicular fluid was decreased.

There seems to be a discrepancy between the results achieved with the Gingival Index and with the gingival crevicular fluid flow. However, it should be stressed that the Gingival Index is assessing inflammation in the marginal gingival tissues and the increase in inflammation in the dressed segments at 2 weeks may be explained by the plaque retentive effect of the dressing and by the direct mechanical irritation produced by the 
Table 4

Mean Differences in Gingival Crevicular Fluid at Different Observation Periods Pairwise t-test

\begin{tabular}{|c|c|c|c|c|c|c|c|c|c|c|}
\hline$N=15$ & Baseline & 2 Weeks & Diff.* & Sign. & 1 Month & Diff.* & Sign. & 2 Months & Diff.* & Sign. \\
\hline \multicolumn{11}{|l|}{ Mesial } \\
\hline Undressed & 5.70 & 6.97 & $+1.27 \dagger$ & \multirow{2}{*}{ NS } & 4.83 & $-0.87 \ddagger$ & \multirow{2}{*}{ NS } & 2.47 & $-3.23 \ddagger$ & \multirow{2}{*}{ NS } \\
\hline Dressed & 5.43 & 6.13 & $-0.70 \ddagger$ & & 3.43 & $-2.00 \ddagger$ & & 3.03 & $-2.40 \ddagger$ & \\
\hline \multirow{2}{*}{$\begin{array}{l}\text { Undressed } \\
\text { Dressed }\end{array}$} & 3.80 & 5.40 & $-1.60 \ddagger$ & \multirow{2}{*}{ NS } & 3.67 & $-0.13 \ddagger$ & \multirow{2}{*}{ NS } & 2.10 & $-1.70 \ddagger$ & \multirow{2}{*}{ NS } \\
\hline & 3.47 & 5.57 & $+2.10 \dagger$ & & 2.77 & $-0.70 \ddagger$ & & 2.70 & $-0.77 \ddagger$ & \\
\hline
\end{tabular}

* Difference from baseline.

$\uparrow$ Denotes increase in GCF.

$\ddagger$ Denotes decrease in GCF.

Table 5

Number and Percentage of Patients Experiencing General Discomfort, Discomfort Upon Eating and Preference for Dressing or No Dressing

\begin{tabular}{lccr}
\hline $\mathrm{N}=15$ & $\begin{array}{c}\text { Discomfort } \\
\text { (general) }\end{array}$ & $\begin{array}{c}\text { Discomfort } \\
\text { (upon eating) }\end{array}$ & Preference \\
\hline Undressed & $33 \%(5)$ & $20 \%(3)$ & $60 \%(9)$ \\
Dressed & $33 \%(5)$ & $20 \%(3)$ & $40 \%(6)$ \\
$\begin{array}{l}\text { Same or no } \\
\text { preference }\end{array}$ & $33 \%(5)$ & $60 \%(9)$ & $0 \%(0)$ \\
\hline
\end{tabular}

pack. Although plaque accumulation in the present study was not evaluated, it has been shown that plaque does accumulate beneath the dressing adjacent to the soft tissues. ${ }^{7-9}$ This could account for this initial increase in gingival inflammation in the dressed segments. Patients began oral hygiene procedures, brushing and flossing, in the undressed segments 12 to 24 hours postoperatively. The reduction of plaque in these segments could account for the decrease in gingival inflammation observed at 2 weeks. As time progressed and oral hygiene procedures were performed in both the dressed and undressed segments, gingival inflammation decreased below baseline levels and the differences between the control and experimental segments were statistically and clinically insignificant.

Gingival crevicular fluid flow on the contrary showed a similar pattern for both the dressed and undressed segments. The increase at 2 weeks may be attributed to the surgical procedure itself. As internal healing progressed a reduction in fluid flow occurred, and at 2 months postoperatively the level was below baseline. The gingival crevicular fluid results were in agreement with those of Greensmith and Wade ${ }^{1}$ and Jones and Cassingham. ${ }^{2}$

According to the questionnaire submitted to each patient, 33\% experienced discomfort without the dressing, $33 \%$ with the dressing, and $33 \%$ observed no difference. The majority of the patients, $60 \%$, preferred no dressing following periodontal surgery while $40 \%$ preferred the dressing. These subjective findings are in agreement with the studies mentioned above, ${ }^{1.2}$ except that they reported increased frequency and severity of discomfort with the use of dressings. In the present study, two patients stated a preference for the dressing, not because of pain but because of a psychological feeling of well being. They stated that the dressing gave them confidence that the surgical area would not be traumatized and that the sutures would not be broken. They admitted that the degree of discomfort upon eating was the same whether a dressing was used or not. In no instances were problems with lost sutures or bleeding encountered when a dressing was not used. It is recognized that the results from this questionnaire have the same drawbacks that other observations obtained with similar subjective procedures.

Clinical Significance. Based on the results of this clinical study, the use of a dressing following modified Widman flap surgery does not improve or hasten healing and its use should therefore be a matter of personal preference. In addition, the use of a dressing does not decrease postoperative discomfort. It must be stressed that in the present study, care was taken to obtain good flap adaptation in all instances. Well adapted flaps might have served as a natural barrier against bacterial penetration. Although plaque scores were not included in the study, regular prophylaxes were performed and oral hygiene was considered a vital part of the study. The results are applicable only to modified Widman flap surgery in which good soft tissue adaptation to the teeth and supporting bone has been achieved. No conclusions can be drawn relative to other surgical procedures where "epithelial protection" is not achieved.

\section{REFERENCES}

1. Greensmith, A. L., and Wade, A. B.: Dressing after reverse bevel flap procedures. J Clin Periodontol 1: 97, 1974

2. Jones, T., and Cassingham, R.: Comparison of healing following periodontal surgery with and without dressings in humans. $J$ Periodontol 50: 387, 1979.

3. Löe, H. and Silness, J.: Periodontal disease in pregnancy I. Prevalence and severity. Acta Odont Scand 21: 533, 1963.

4. Burgett. F. G., Knowles, J., Nissle, R., Shick, R., and Ramfjord, S.: Short term results of three modalities of periodontal treatment. $J$ Periodontol 48: 131, 1977.

5. Abbott, B., and Caffesse, R.: Crevicular fluid: origin, composition, methods of collection and clinical significance. $J$ West Soc Periodontol 25: 164, 1977.

6. Hancock, E., Cray, R., and O'Leary, T.: The relationship between gingival crevicular fluid and gingival inflammation. $J$ Periodontol 50: 13, 1979. 
7. Heaney, F. G., and Appleton, J.: The effect of periodontal dressings on the healthy periodontium. J Clin Periodontol 3: 66, 1976.

8. Pihlstrom, B. L., Thorn, H. L., and Folke, L. E.: The effect of periodontal dressing on supragingival microorganisms. J Periodontol 48: 440, 1977.

9. Plüss, E. M., Engelberger, P., and Rateitschak, K.: Effect of chlorhexidine on dental plaque formation under periodontal pack. $J$ Clin Periodontol 48: 566, 1977.

Send reprint requests to: Dr. Raul G. Caffesse, University of Michigan School of Dentistry, Department of Periodontics, Ann Arbor, MI 48109.

\section{Announcements}

\section{THE UNIVERSITY OF PENNSYLVANIA SCHOOL OF DENTAL MEDICINE}

The University of Pennsylvania School of Dental Medicine announces the following courses:

Titi.f: Periodontal Prosthesis: The Five-Day Program (in Six)

DATE: October 23-28, 1983

FACUltY: Morton Amsterdam, D.D.S., ScD., Course Director, Professor of Periodontics, University of Pennsylvania and D. Walter Cohen, D.D.S., ScD., Professor of Periodontics and faculty

This extensive and intensive program has been presented annually for over 25 years. The Five-Day Program is offered only at Penn. This most comprehensive course is designed to correlate periodontal therapeutics and advanced restorative procedures.

Topics covered include: etiology and pathology of periodontal disease; initial preparation for the periodontal case; mucogingival and flap surgery; osseous regeneration procedures: the evaluation and treatment of pathologic occlusion; principles, techniques and hazards of orthodontics for the periodontal case; diagnosis and overall treatment planning-sequence of therapy; bite plane therapy-occlusal adjustment; splinting-indications and contraindications; tooth preparation and provisional restorations; fixed, removable and telescopic restorations; repair and maintenance.

TituE: Balancing Body Chemistry

D^TE: October 6-9, 1983

FACULTY: Kenneth Fordham, D.D.S., Associate in Periodontics, University of Pennsylvania

Without proper nutrition and dietary supplementation the success of treatment may be limited or compromised. Balancing body chem- istry is intended to be an adjunct to routine patient care, while in some cases it may eliminate the need for surgical intervention. Pioneered by Dr. Melvin Page, this approach to health care has proven successful for more than 50 years.

In this course, the faculty will discuss how to prevent illness in patients. A balanced body chemistry is vital to the health of all people, and participants will have their own body chemistries analyzed during the course.

TIrı.E: The Most Important Prescriptions in Dental Practice Date: September 23, 1983

FACULTY: Sam V. Holroyd, D.D.S., M.S. (Pharmacology), MS (periodontics), FACD, FICD, Professor and Chairman Department of Periodontics and Course Director in Pharmacology, School of Medicine, Washington University, St. Louis, Missouri

This 1-day course will briefly review the general principles of drug administration and then consider the drugs most frequently prescribed in dental practice. The clinical characteristics which will allow the dentist to select one agent over another will be reviewed. This will be followed by a discussion of examples of prescriptions which will cover essentially all dental indications. Antibiotics, analgesics, sedative, tranquilizers, antisialogogues, nutritional aids and various medications used in the treatment of common oral disease entities will be discussed. A handout which includes prescription examples will be used to facilitate discussion. The most îrequently prescribed drugs in medical practice will also be discussed from the standpoint of dental implications.

For further information contact: Eileen Bliss. Assistant Director. University of Pennsylvania Dental School Continuing Education, 4001 Spruce Street, Philadelphia, PA 19104. (215) 898-6505.

\section{THE NORTHEASTERN SOCIETY OF PERIODONTISTS}

The Northeastern Society of Periodontists announces The Fall Meeting.

DATE: $\quad$ Friday, October 21, 1983

PlACE: $\quad$ New York Sheraton Hotel, 55th St and Seventh Ave, New York, NY

Presentation: "Orthodontic-Periodontic Dilemma"

SPEAKERS: Drs. J. Gary Maynard, Jr. and Arnold M. Geiger

For further information please contact: Irwin W. Scopp, D.D.S., Veterans Administration Medical Center, 408 First Ave. New York, NY 10010. 